\title{
The Motional EMF in Conductors with Ferromagnetic Shield
}

\author{
Alexander I. Korolev \\ Russian State Scientific Center for Robotics and Technical Cybernetics (RSSC RTC), St. Petersburg, Russia \\ Email: a.korolev@rtc.ru
}

How to cite this paper: Korolev, A.I. (2018) The Motional EMF in Conductors with Ferromagnetic Shield. Open Access Library Journal, 5: e4461. https://doi.org/10.4236/oalib.1104461

Received: March 1, 2018

Accepted: March 24, 2018

Published: March 27, 2018

Copyright (c) 2018 by author and Open Access Library Inc.

This work is licensed under the Creative Commons Attribution International License (CC BY 4.0).

http://creativecommons.org/licenses/by/4.0/

\begin{abstract}
The motional EMF in segments of the copper wire with magnetic shielding is found according to the voltage lack in two coils with partial shielding moving relative to the magnetic field lines. The first coil moved across the Earth's magnetic field lines. The second one was located near the end face of the rotating disk magnet with an axial magnetization. Permalloy foil wound around a part of turns containing wire was used for shielding. The experiments result reveals the penetrating ability of the magnetic field through the ferromagnetic shield and shows the physical nature of the superposition principle. With this in mind, a universal method for calculating EMF induced in a conductive body has been provided as well as the concepts of magnetic field lines velocity and acceleration have been introduced.
\end{abstract}

\section{Subject Areas}

Applied Physics, Experimental Physics

\section{Keywords}

Motional EMF, Magnetic Shielding, Electromagnetic Induction

\section{Introduction}

In magnetostatics, it is believed that with a passive magnetic shielding the magnetic field flow is attracted into the magnetic shield area. Magnetic field practically does not penetrate [1] [2] [3] [4] inside the sufficiently well shielded cavity. However, such concept of magnetic shielding does not correspond physically to the principle of superposition. Therefore, the latter is understood only as a mathematical technique convenient for calculating the magnetic field rather than as a result of the physical superposition of elementary magnetic fields at a point. The concept of the magnetic field absence in the shielded cavity has led to the 
patenting of the device for measuring the moving object speed according to the sum of the motional EMFs both in the conductor's segment with magnetic shield and without it [5]. In the authors' idea, the total induced EMF should be caused by the motion of partially shielded coil with a wire across the Earth's magnetic field lines. But in the work [6], there is no information about magnetic shield impact on the motional EMF value. In this work, the attempt to measure the velocity using the way [5] is made. An experiment according to the scheme close to that one described in the work [6] was also conducted. During that experiment, the motional EMF was measured in the partially shielded coil with a wire placed near the end face of the wide rotating magnet. The experiments result allows a new look at a magnetic field properties such as penetrating ability, additivity, and mobility of the field lines. The universal method for calculation the EMF induced in a conductor according to the known picture of the magnetic field lines motion is presented.

\section{Description of Experiments}

\subsection{Experiment with the Earth' Magnetic Field}

Figure 1 shows the experimental design on measuring the speed of the partially shielded coil with the wire. Frame 1 is made of the square aluminum profile of $15 \mathrm{~mm}$ at width, bent as a large square with a length of the inner side of $100 \mathrm{~mm}$. Inside the frame, $610 \mathrm{~m}$ of enameled copper wire with a diameter of $0.2 \mathrm{~mm}$ is laid. Approximate number of turns: 1385 . For additional protection, the insulating tape is wound on the frame. On one side of obtained coil, the permalloy foil 2 of $100 \mathrm{~mm}$ at width and $20 \mu \mathrm{m}$ in thickness is wound. Winding is a thin-walled cylinder with a diameter of $22 \mathrm{~mm}$ and 10 layers of foil. To measure the voltage in the coil, the multimeter is used in the measurement mode of constant voltage with a precision of $1 \mathrm{mV}$. The coil moves perpendicular to the frame plane at speed $v=90 \pm 10 \mathrm{~km} / \mathrm{h}$. The vector projection of the Earth's magnetic induction on the frame plane $\boldsymbol{B}$ consists of the horizontal $\boldsymbol{B}_{\boldsymbol{x}}$ and vertical $\boldsymbol{B}_{\boldsymbol{y}}$ components. Magnitude of the horizontal intensity of the Earth's magnetic field at the place of observation (St. Petersburg) is $H_{g} \simeq 15 \mu \mathrm{T}$ according to [7]. The angle between this component and $\boldsymbol{B}_{\boldsymbol{x}}$ equals to 20.4. Thus, the magnitude of $\boldsymbol{B}_{\boldsymbol{x}}$ can be calculated according to the formula (1):

$$
B_{x}=\mu H_{g} \cos \left(20.4^{\circ}\right) \cong 14 \mu \mathrm{T}
$$

Here $\mu \simeq 1$ is the magnetic permeability of the air atmosphere.

The experiment was carried out inside a moving vehicle. The weakening of the magnetic field by the vehicle's steel body at the place of measuring (near the window) was not significant, since the compass with a magnetic arrow operated like outside the vehicle's body as well.

In coil sides, the motional EMF caused by the Lorentz forces acting on the free electrons of the conductor appears. Lorentz force $F_{L y}$ acting along the upper and lower sides of the loop are mutually compensated. Lorentz forces along the sides $F_{L x}$ according to the existing theory, are not to be compensated due to the 


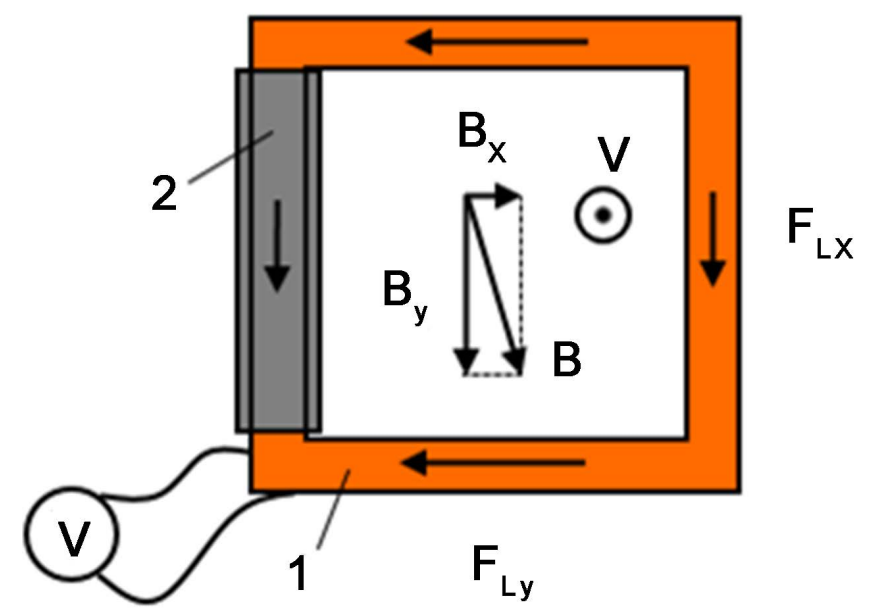

Figure 1. Scheme of experiment No. 1.

shielding of the magnetic field on the side with permalloy shield. The shielding effectiveness of the thin-walled cylinder from $N$ layers of foil can be evaluated [1] by formula (2):

$$
T=\frac{D}{\mu N d}
$$

Here $T$ is the coefficient of magnetic field attenuation inside the cylinder, $\mu \geq 50000$ is the coefficient of magnetic permeability of permalloy, $D$ is the cylinder diameter, $d$ is the foil thickness. Thus, $T \leq 0.02$. Taking into account the edge effects, the attenuation coefficient should be slightly higher. The amount of EMF in the coil sides that should be indicated by voltmeter can be calculated according to the formula (3):

$$
\varepsilon_{i \Sigma}=(1-T) \cdot v \cdot B_{x} \cdot l \cdot n
$$

Here $l$ is the width of shielding foil, $n$ is the number of wire turns. Under the conditions described, a voltage indication can be expected on the voltmeter $\varepsilon_{i \Sigma} \simeq 47 \mathrm{mV}$.

\subsection{Experiment with Magnetic Field of the Disc Magnet}

Figure 2 provides a diagram of the experiment to measure the motional EMF in the partially shielded coil located in the field of the rotating permanent magnet. On the steel channel profile 1, the DC electric motor 2 with rated power of 12.2 $\mathrm{W}$ is secured. The motor starts from the power source when the voltage is $12 \mathrm{~V}$. On the engine shaft, the brass cone 4 is suspended using the polypropylene rope 3. The cone is bonded to the end face of the disc magnet 5 with axial magnetization. The magnet radius $R=25 \mathrm{~mm}$, thickness $D=10 \mathrm{~mm}$.

The magnet is made of $\mathrm{NdFeB}$ with the amount of retained magnetization, according to the description, $B_{r}=1.44-1.48 \mathrm{~T}$. The value of residual magnetization was determined after measuring the magnetic field near the magnet end face using the Hall sensor. The calculation according to [8] can be carried out by the formula (4): 


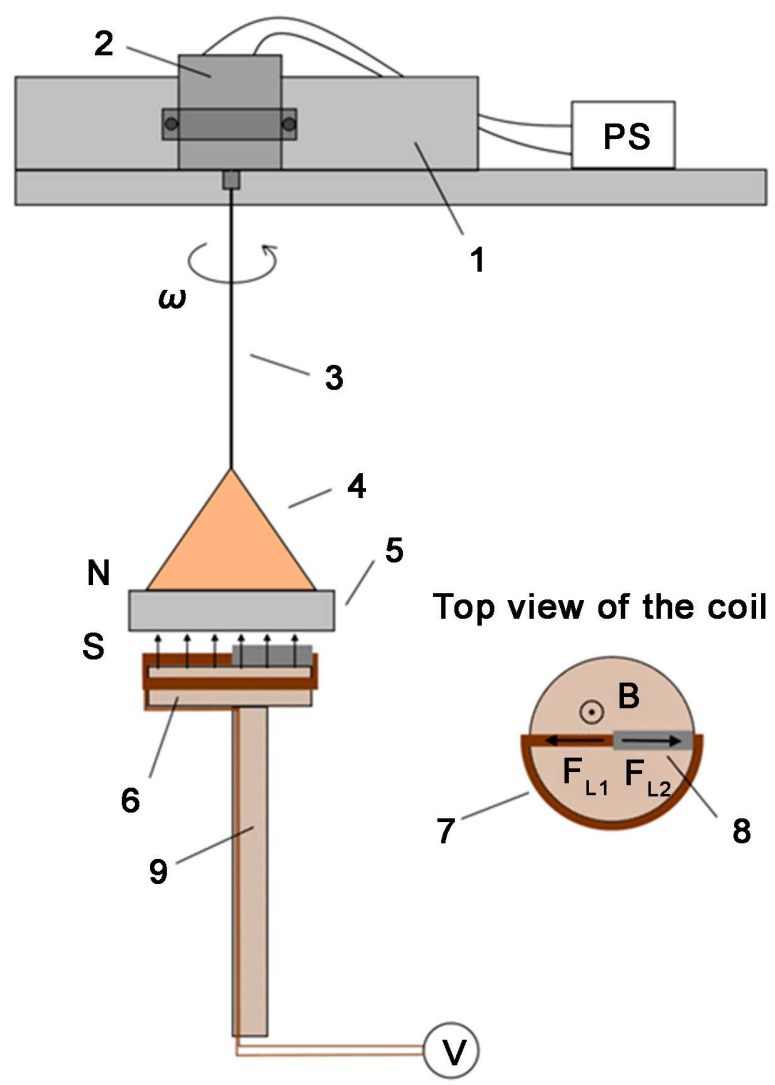

Figure 2. Scheme of experiment No. 2.

$$
B_{r}=2 B_{z}\left(\frac{D+z}{\sqrt{R^{2}+(D+z)^{2}}}-\frac{z}{\sqrt{R^{2}+z^{2}}}\right)^{-1}
$$

Here $B_{z}$ is the magnitude of magnetic induction on the magnet axis at a distance from the end face $\mathrm{z}$. The measured value at $\mathrm{z}=20 \mathrm{~mm}$ was $908 \mathrm{Gs}$, which corresponds to the magnitude of $B_{r} \simeq 1.27 \mathrm{~T}$. The magnet is revolved by motor at angular velocity $\omega \simeq 425 \mathrm{rad} / \mathrm{s}$. To determine the angular velocity, the photographing of rotating magnet with two marker nuts secured diametrically opposite on the magnet sidewall was used. With the ambient lighting, the nuts leave shiny tracks, see photo on Figure 3. $\omega$ is defined as the ratio of shiny segment angle $(1.06 \mathrm{rad})$ to holding time of frame $(1 / 400 \mathrm{~s})$.

The textolite disk 6 of radius $R^{*}=20 \mathrm{~mm}$ and thickness of $15 \mathrm{~mm}$ is brought to the rotating magnet from below. The coil 7 consisting of $n=10$ copper wire turns with cross-section of $0.03 \mathrm{~mm}^{2}$ in fluoroplastic insulation is secured to the disc. The external part of the coil is located along the disc circumference, the internal-along its diameter. One radial part of the coil is placed in the shielding cavity 8 . The cavity is constructed in the form of cylinder $5 \mathrm{~mm}$ in diameter containing 15 layers of permalloy foil with a thickness of $20 \mu \mathrm{m}$. The cylinder is unevenly flattened due to its securing to the disc with the help of Scotch tape. The maximum ratio of the ellipses' semiaxes lengths in the resulting cavity 


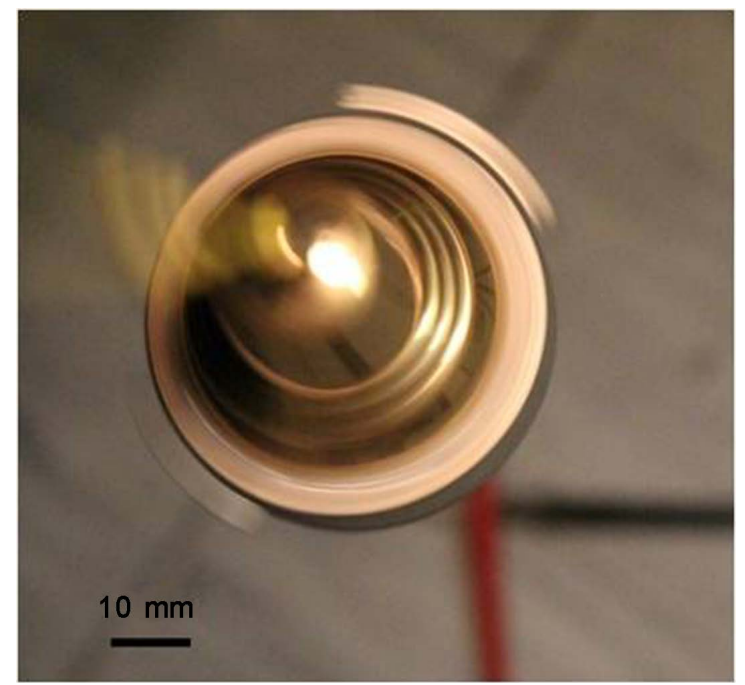

Figure 3. The magnet is in rotation with two marker nuts on its sidewall. Holding time of the frame is $1 / 400 \mathrm{~s}$.

section reaches two. The evaluation of the magnetic field attenuation coefficient inside the cavity according to the formula (2) gives a value $T \simeq 0.00033$. For ease of use, the textolite disc is provided with a handle in the form of wooden rod 9. The coil terminals are arranged along the magnet axis. The coil voltage is measured by multimeter in voltmeter mode with accuracy of $1 \mathrm{mV}$.

When approaching the coil to rotating magnet from below, the permalloy shield is magnetized, and the attractive force appears. This force destabilizes the magnet rotation. To reduce the magnet beating in the vertical direction, it was slightly directed by the side hand touch. With this, rotation speed reduced and the reduction magnitude could be measured by changing the tone of the engine sound. By sound, this reduction did not exceed two times, that is $\omega \geq 200$ $\mathrm{rad} / \mathrm{s}$. With the distance between the coil diametric part and the magnet surface of the $10 \mathrm{~mm}$ the beats did not exceed $\pm 2 \mathrm{~mm}$. In this case, estimation of the magnetic field magnitude in the area of the coil diametric part made according to the formula (4) gives a value of $B \simeq 0.16 \mathrm{~T} \mathrm{~T}$. When rotating the magnet, in the coil two radial Lorentz forces appears: $F_{L 1}$ и $F_{L 2}$, one of which, due to magnetic shielding, might be close to zero. The amount of the motional EMF induced in the radial parts of the coil can be estimated by the formula (5):

$$
\varepsilon_{i \Sigma}=(1-T) \cdot \bar{v} \cdot B \cdot R^{*} \cdot n
$$

Here $\bar{v}=\frac{1}{2} \omega R^{*}$ is the mean linear velocity of the relative motion of the coil radial parts and the magnetic field lines. The voltage indication can be expected on the voltmeter $\varepsilon_{i \Sigma} \geq 64 \mathrm{mV}$.

\section{Result and Discussion}

The result of the voltage measurements in both experiments is the same. The measured value is $0 \pm 1 \mathrm{mV}$. Possible sources of the measurement errors are: 
Heterogeneity of the magnetic field, the edge effects near the end faces of the shielding cavities, the inaccuracy of the coils orientation relative to magnetic field lines, gaps between the layers of permalloy foil, real value of the permalloy magnetic permeability coefficient different from the reference one. These all can not impact significantly during the measurement of voltages with a precision of $2 \%$ for experiment No.1 and 1.5\% for experiment No.2.

The result obtained is explained with the presence of the motional EMF in the conductors located inside the shielded cavities. With this, the magnitude of this EMF coincides accurately with those one in the unshielded sections of the conductors. This indicates the penetrating ability of the magnetic field through the ferromagnetic shield. Thus, the superposition principle takes the physical meaning. The magnetic field at a point is a result of physical superposition of the elementary magnetic fields from individual dipoles including the induced ones. Such representation of the superposition principle is, for example, here [9]. Magnetic shielding is known to protect the conductor from the usual induced EMF while in the external alternating magnetic field. Why it does not protect against motional EMF?

Work [10] introduces the concept of "magnetic force" acting on the charge located in the alternating magnetic field of some elementary source-dipole. If consider the effect of such forces to the shielded conductor in an alternating magnetic field taking into account the principle of superposition, one can find that they mutually compensate each other. Each displacement of the magnetic field lines from external source corresponds to the opposite displacement of the secondary magnetic field lines from the magnetized shield. Such compensation does not occur for the motional EMF because the lines of the magnetic field source and the shield move with different speeds relative to the conductor (some of the move, others are motionless).

If remember the classical picture of magnetic field lines as the streamlines of luminiferous medium, one can give the universal method for calculating the electromagnetic induction that occurs in a conductive body. The correctness of such approach is confirmed, for example, in [11]. The general principle of the calculation was given by M. Faraday while observing EMI in conductors of various shapes [12]. Namely: EMF is induced in a conductor when crossing its with magnetic streamlines. It is now known that charged particles exhibit magnetism because of their own angular momentum (spin). There is an obvious analogy between the EMI effect and the Magnus effect in mechanics. Therefore, the force acting on the charge $q$ in the magnetic field can be represented in form (6):

$$
F_{m}=q \sum_{i=1}^{n}\left[v_{i}, B_{i}\right]
$$

Here $\boldsymbol{B}_{\boldsymbol{i}}$ is a vector of magnetic induction generated at a charge location point by the elementary field source with number $i . v_{i}$ is velocity vector of the charge movement relative to the field lines of $i$ source. For the sake of practical calculations, the amount can be replaced with a spatial integral in terms of the source 
volume provided that its size $\gg$ of the elementary magnetic dipoles. $\boldsymbol{V}_{\boldsymbol{i}}$ can be represented as amount (7):

$$
v_{i}=v_{i \tau}+v_{i r}
$$

Here $\boldsymbol{v}_{\boldsymbol{i} \tau}$ is a vector of peripheral component of velocity that is tangential to surface with $B_{i}=$ Const.$v_{i r}$ is a vector of radial component that is directed perpendicular to the surface. The magnitude of the latter can be found in the formula (8):

$$
v_{i r}=\frac{1}{\left|\nabla B_{i}\right|} \cdot \frac{\partial B_{i}}{\partial t}
$$

The expression for induced EMF in [10] was given without taking into account the gradient of magnetic induction. Without this, a different number of lines (flux) can pass through a turn per unit time. A more precise expression including the Lorentz force and the classical law of EMI, can be obtained by integrating $F_{m}$ having effect on a single charge along some path $L$ between the measurement points of potential (9).

$$
\varepsilon_{\text {ind }}=-\frac{1}{q} \int_{L}\left(F_{m}, \mathrm{~d} l\right)
$$

The independence of $\varepsilon_{\text {ind }}$ on the integration path is true provided that there is a homogeneous magnetic field moving at a fixed speed in the conductive body under study. If this is not the case, i.e. the pictures of the charges movement relative to the field lines in various paths are different, it is necessary to break the body into small segments, calculate the force in each segment according to the formula (6) then make integration according to the formula (9) by all possible paths of current. Then the resulted array of $\varepsilon_{\text {ind }}$ is added according to a known addition formula of EMF sources (paths) subject to their electrical resistance. The calculation of $\varepsilon_{\text {ind }}$ becomes much simpler when considering the thin conductor segment. In this case, the integration is carried out by its spatial curve.

During the charge movement in the homogeneous magnetic field, the addition of the force tangential components $F_{m}$ will result in the Lorentz force. With this, the radial components are mutually compensated. Further integration will provide the clear motional EMF.

The charges movement in the inhomogeneous alternating magnetic field across the magnetic field lines is implemented, for example, in the standard problem when placing a wire turn inside the long solenoid, symmetrically to the axis of the latter. Induced EMF in the turn is caused by the alternating magnetic field of the solenoid and is calculated according to the faraday's law of electromagnetic induction. In the law, the flux rate change of the magnetic induction passing through the turn corresponds to the expression (9) with the substitution (8).

$\boldsymbol{V}_{\boldsymbol{i r}}$ can be expressed via derivative of magnetic induction (8). $\boldsymbol{V}_{\boldsymbol{i} \tau}$ is not expressed via $B_{i}$. This points to the necessity of introducing such a parameter of the magnetic field as the displacement velocity of the magnetic field lines $\boldsymbol{V}_{B}$. In a 
static case, it will be zero. In a dynamic case, the three-dimensional picture of $\boldsymbol{V}_{\boldsymbol{B}}$ distribution together with a picture of magnetic field lines will give a more complete description of the magnetic field in some area. Due to the fact that the magnetic field has a finite rate of propagation, it is better to call it "magnetic flow" when considering fast processes. Streamlines slipping and rotation of these flows can be characterized by the distribution of $\boldsymbol{V}_{\boldsymbol{B}}$ velocity and, if necessary, by acceleration of $\boldsymbol{a}_{B}$ at any time. At periodic movements it can be said about the expansion of "magnetodynamic waves" [13].

\section{Conclusions}

During two experiments, the presence of motional EMF inside the ferromagnetic shields made of permalloy foil has been detected. Magnetic coils with partially shielding windings and placed in homogeneous magnetic fields were used. During the first experiment, the coil moved relative to the Earth's magnetic field, and during the second one, the magnetic field lines of the rotating disk magnet moved relative to the coil. The lack of voltage on the coils terminals indicates with a good accuracy the presence of motional EMF in the shielded parts of windings equal in magnitude to those ones in parts without a shield. This reveals the penetrating ability of the magnetic field through the ferromagnetic shield and shows the physical nature of the superposition principle. As a result, the universal method for calculating the induced EMF that occurs in the conductive body moving relative to magnetic field was represented. It withdraw the paradoxes in calculating induced EMF on the classical law [14] and shows the conditionality of dividing induced EMF into the usual and "motional" part. For the description of dynamic magnetic fields, it is necessary to introduce the kinematic parameters of the movement of their field lines, such as speed and acceleration. These parameters can be determined experimentally by introducing two closely spaced conductive probes into the region of the magnetic field under study. The tips of the probes should be placed with a slight shift in a direction perpendicular to the magnetic power lines. The difference in motional EMF in the probes can be registered using a voltmeter with high input impedance. The dynamic magnetic field of permanent magnets rotating around their own magnetization axes was registered in [15] [16]. To do this, the authors used cylindrical capacitors arranged symmetrically to the axes. The potential difference that appears on the plates is explained by the action of uncompensated Lorentz forces in the lead wires. The advantage of this method for detecting a rotating magnetic field in comparison with the introduction of probes is the availability of essentially larger capacitance, which reduces the requirement for input impedance of the voltmeter. However, for characteristic of a moving magnetic field with complex configuration in a point, thin probes are required.

Awareness of motional EMF presence inside the shielded cavity can be used in the technique of, for example, the shielded signal cables laying in fast-moving vehicles. The nonequivalence of the rotating magnetic field to the stationary one 
with the fixed picture of the field lines is the cause of the EMF emerges in the external circuit of unipolar generator when the disk rotates along with the magnet [17] [18].

\section{References}

[1] Yashchuk, V., Lee, S. and Paperno, E. (2013) Magnetic Shielding. In: Budker, D. and Jackson Kimball, D., Eds., Optical Magnetometry, Cambridge University Press, Cambridge, 225-248. https://doi.org/10.1017/CBO9780511846380.013

[2] Tesche, F.M., Ianoz»M. and Karlsson, T. (1997) EMC Analysis Methods and Computational Models. Wiley-Interscience, p. 510.

[3] Tsaliovich, A. (2012) Cable Shielding for Electromagnetic Compatibility. Springer Science \& Business Media, p. 261.

[4] Keshtkar, A., Maghoul, A. and Kalantarnia, A. (2011) Magnetic Shield Effectiveness in Low Frequency. International Journal of Computer and Electrical Engineering, 3, 507-513. https://doi.org/10.7763/IJCEE.2011.V3.370

[5] Korovin, V. and Korovin, K. (2007) Patent RU 2307 356C1, G01P3/50, Pub. date 27.09.

[6] Guala-Valverde, J., Blas, R. and Mazzoni, P. (2006) Non Local Motional Electrodynamics. Annales de la Fondation Louis de Broglie, 31, 75-91.

[7] Smirnova, T. (2008) Magnitnoe pole Zemly: Opredelenie modulja gorizontal'noi sostavljajuschei naprjazhennosty geomagnitnogo polja: Ucheb. Method. Posobie/ NovGU, Velikiy Novgorod, 49 p.

[8] Camacho, J.M. and Sosa, V. (2013) Alternative Method to Calculate the Magnetic Field of Permanent Magnets with Azimuthal Symmetry. Revista Mexicana de Física E, 59, 8-17.

[9] Rozin, M. and Budjak, D. (2018) Magnitnoje pole v veschestve. http://www.rosinmn.ru/ELEKTRO_magnet_3.htm

[10] Korolev, A.I. (2013) On Electromagnetic Induction in Electric Conductors, arXiv:1303.0785 [physics.gen-ph].

[11] Fitzpatrick, R. (2017) Electromagnetism and Optics (An Introductory Course). http://farside.ph.utexas.edu/teaching/3021/lectures/node88.html

[12] Faraday, M. (1947) Experimental Investigation on Electricity, v. 1. ed. Academy of Science of the USSR, Moscow.

[13] Korolev, A.I. (2013) Magnetodynamic Waves in the Air. JMMM, 327, 172-176. https://doi.org/10.1016/j.jmmm.2012.09.005

[14] Nussbaum, A. (1972) Faraday's Law Paradoxes. Physics Education, 7, 231. https://doi.org/10.1088/0031-9120/7/4/006

[15] Tymofeev, V. and Tymofeeva, T. (2012) Nekotorye svoistva electricheskogo polja magnitnogo rotator. Vestnik SVFU.

[16] Misuchenko, I. (2014) Eksperimental'noe issledovanie elektricheskogo polja vraschajuschegosja vokrug osi namagnichennosti kol'cevogo magnita.

http://electricaleather.com/d/358095/d/elektricheskoe-pole-vraschayuschegosya-kol cevogo-magnita.pdf

[17] Kelly, A.G. (2004) Experiments on Unipolar Induction. Annales de la Fondation Louis de Broglie, 29, No. 1-2.

[18] Kann, K. (2018) Strannosti unipoljarnoi induktsii. http://docplayer.ru/27574165-Strannosti-unipolyarnoy-indukcii-k-b-kann.html 